\title{
Correction to: Update of the Culicoides (Diptera: Ceratopogonidae) species checklist from Algeria with 10 new records
}

Mounira Belkharchouche ${ }^{1,2,3,4^{*}}$, Selima Berchi ${ }^{3}$, Bruno Mathieu ${ }^{5}$, Ignace Rakotoarivony ${ }^{4,6}$, Maxime Duhayon $^{4,6}$, Thierry Baldet ${ }^{6,7}$ and Thomas Balenghien ${ }^{6,8,9^{*}}$

\section{Correction to: Parasites Vectors (2020) 13: 463} https://doi.org/10.1186/s13071-020-04335-4

Following publication of the original article [1], it was brought to the authors' attention that Table 1 had published with incomplete information.
The published article has since been updated and the corrected table may be found in this correction.

The authors apologize for any inconvenience caused.

The original article can be found online at https://doi.org/10.1186/s13071020-04335-4.

*Correspondence: mimibel7425@gmail.com; thomas.balenghien@cirad.fr

${ }^{1}$ Ecole Nationale Supérieure de Biotechnologie, Taoufk Khaznadar,

nouveau pôle universitaire Ali Mendjeli, B.P. E66, 25100, Constantine,

Algérie

${ }^{6}$ ASTRE, University of Montpellier, CIRAD, INRAE, Montpellier, France

Full list of author information is available at the end of the article

(c) The Author(s) 2021. This article is licensed under a Creative Commons Attribution 4.0 International License, which permits use, sharing, adaptation, distribution and reproduction in any medium or format, as long as you give appropriate credit to the original author(s) and the source, provide a link to the Creative Commons licence, and indicate if changes were made. The images or other third party material in this article are included in the article's Creative Commons licence, unless indicated otherwise in a credit line to the material. If material is not included in the article's Creative Commons licence and your intended use is not permitted by statutory regulation or exceeds the permitted use, you will need to obtain permission directly from the copyright holder. To view a copy of this licence, visit http://creativeco mmons.org/licenses/by/4.0/. The Creative Commons Public Domain Dedication waiver (http://creativecommons.org/publicdomain/ zero/1.0/) applies to the data made available in this article, unless otherwise stated in a credit line to the data. 
Table 1 List of the material examined to produce 10 new records for the Algerian fauna

\begin{tabular}{|c|c|c|c|c|c|c|}
\hline Species & Type of material & Country & Locality & Coordinates & Date & Material examined \\
\hline \multirow{7}{*}{$\begin{array}{l}\text { Culicoides (Avaritia) chiop- } \\
\text { terus (Meigen) }\end{array}$} & \multirow[t]{7}{*}{ Non-type material } & Algeria & Ain El Deheb & $34^{\circ} 51^{\prime} \mathrm{N}, 1^{\circ} 29^{\prime} \mathrm{E}$ & 23-24 Mar 2018 & 1 female \\
\hline & & \multirow[t]{6}{*}{ France } & Andevanne & $49^{\circ} 23^{\prime} \mathrm{N}, 5^{\circ} 4^{\prime} \mathrm{E}$ & 17-18 Oct 2006 & 1 female \\
\hline & & & Caro & $43^{\circ} 8^{\prime} \mathrm{N}, 1^{\circ} 14^{\prime} \mathrm{W}$ & 23-24 Oct 2006 & 1 female \\
\hline & & & Nuillé sur Vicoin & $47^{\circ} 57^{\prime} \mathrm{N}, 0^{\circ} 45^{\prime} \mathrm{W}$ & 4-5 Jul 2011 & 1 female \\
\hline & & & Orbey & $48^{\circ} 7^{\prime} \mathrm{N}, 7^{\circ} 7^{\prime} \mathrm{E}$ & 25-26 May 2009 & 5 females \\
\hline & & & Pontivy & $48^{\circ} 4^{\prime} \mathrm{N}, 2^{\circ} 58^{\prime} \mathrm{W}$ & 18-19 Jan 2012 & 5 females \\
\hline & & & Schwobsheim & $48^{\circ} 13^{\prime} \mathrm{N}, 7^{\circ} 34^{\prime} \mathrm{E}$ & 18-19 Apr 2011 & 1 female \\
\hline \multirow{11}{*}{$\begin{array}{l}\text { Culicoides (Avaritia) dewulfi } \\
\text { Goetghebuer }\end{array}$} & \multirow[t]{11}{*}{ Non-type material } & \multirow[t]{8}{*}{ Algeria } & Tiaret & $35^{\circ} 23^{\prime} \mathrm{N}, 1^{\circ} 21^{\prime} \mathrm{E}$ & 16-19 Sep 2017 & 1 female \\
\hline & & & Bougara & $35^{\circ} 29^{\prime} \mathrm{N}, 1^{\circ} 55^{\prime} \mathrm{E}$ & 13-15 Nov 2016 & 1 female \\
\hline & & & Sougueur & $35^{\circ} 09^{\prime} \mathrm{N}, 1^{\circ} 31^{\prime} \mathrm{E}$ & 13-16 May 2016 & 2 females \\
\hline & & & Ain El Deheb & $34^{\circ} 51^{\prime} \mathrm{N}, 1^{\circ} 29^{\prime} \mathrm{E}$ & 25-27 Jul 2017 & 3 females \\
\hline & & & Ain El Deheb & $34^{\circ} 51^{\prime} \mathrm{N}, 1^{\circ} 29^{\prime} \mathrm{E}$ & 1-2 Jun 2018 & 1 female \\
\hline & & & Hammadia & $35^{\circ} 26^{\prime} \mathrm{N}, 1^{\circ} 53^{\prime} \mathrm{E}$ & 2-5 Jun 2016 & 1 female \\
\hline & & & Takhmaret & $35^{\circ} 06^{\prime} \mathrm{N}, 0^{\circ} 42^{\prime} \mathrm{E}$ & 12-15 Sep 2017 & 1 female \\
\hline & & & Takhmaret & $35^{\circ} 06^{\prime} \mathrm{N}, 0^{\circ} 42^{\prime} \mathrm{E}$ & 13-14 Sep 2018 & 3 females \\
\hline & & \multirow[t]{3}{*}{ France } & Caro & $43^{\circ} 84 \mathrm{~N}, 1^{\circ} 14^{\prime} \mathrm{W}$ & 29-30 May 2006 & 2 females \\
\hline & & & Cuguen & $48^{\circ} 26^{\prime} \mathrm{N}, 1^{\circ} 39^{\prime} \mathrm{W}$ & 14-15 Nov 2011 & 1 female \\
\hline & & & Lazer & $44^{\circ} 21^{\prime} \mathrm{N}, 5^{\circ} 51^{\prime} \mathrm{E}$ & 18-19 Apr 2011 & 2 females \\
\hline \multirow{30}{*}{$\begin{array}{l}\text { Culicoides (Beltranmyia) } \\
\text { navaiae Lane }\end{array}$} & \multirow[t]{30}{*}{ Non-type material } & \multirow[t]{28}{*}{ Algeria } & Tiaret & $35^{\circ} 23^{\prime} \mathrm{N}, 1^{\circ} 21^{\prime} \mathrm{E}$ & 3-6 Jun 2017 & 2 females \\
\hline & & & Tiaret & $35^{\circ} 23^{\prime} \mathrm{N}, 1^{\circ} 21^{\prime} \mathrm{E}$ & 28-30 Sep 2017 & 1 male, 2 females \\
\hline & & & Tiaret & $35^{\circ} 23^{\prime} \mathrm{N}, 1^{\circ} 21^{\prime} \mathrm{E}$ & $21-22$ Sep 2018 & 2 females \\
\hline & & & Bougara & $35^{\circ} 29^{\prime} \mathrm{N}, 1^{\circ} 55^{\prime} \mathrm{E}$ & 9-12 May 2016 & 3 females \\
\hline & & & Bougara & $35^{\circ} 29^{\prime} \mathrm{N}, 1^{\circ} 55^{\prime} \mathrm{E}$ & 10-13 Nov 2016 & 3 females \\
\hline & & & Bougara & $35^{\circ} 29^{\prime} \mathrm{N}, 1^{\circ} 55^{\prime} \mathrm{E}$ & 15-19 Apr 2017 & 5 females \\
\hline & & & Bougara & $35^{\circ} 29^{\prime} \mathrm{N}, 1^{\circ} 55^{\prime} \mathrm{E}$ & 29-30 Jun 2018 & 1 female \\
\hline & & & Sougueur & $35^{\circ} 09^{\prime} \mathrm{N}, 1^{\circ} 31^{\prime} \mathrm{E}$ & 3-6 Feb 2016 & 11 females \\
\hline & & & Sougueur & $35^{\circ} 09^{\prime} \mathrm{N}, 1^{\circ} 31^{\prime} \mathrm{E}$ & 23-26 Jun 2017 & 4 females \\
\hline & & & Sougueur & $35^{\circ} 09^{\prime} \mathrm{N}, 1^{\circ} 31^{\prime} \mathrm{E}$ & 14-15 Sep 2018 & 2 females \\
\hline & & & Ain El Deheb & $34^{\circ} 51^{\prime} \mathrm{N}, 1^{\circ} 29^{\prime} \mathrm{E}$ & 2-5 Apr 2016 & 11 females \\
\hline & & & Ain El Deheb & $34^{\circ} 51^{\prime} \mathrm{N}, 1^{\circ} 29^{\prime} \mathrm{E}$ & 24-27 Apr 2017 & 2 females \\
\hline & & & Ain El Deheb & $34^{\circ} 51^{\prime} \mathrm{N}, 1^{\circ} 29^{\prime} \mathrm{E}$ & 21-22 Sep 2018 & 27 females \\
\hline & & & Ksar Chellala & $35^{\circ} 15^{\prime} \mathrm{N}, 2^{\circ} 18^{\prime} \mathrm{E}$ & 13-16 May 2016 & 20 females \\
\hline & & & Ksar Chellala & $35^{\circ} 15^{\prime} \mathrm{N}, 2^{\circ} 18^{\prime} \mathrm{E}$ & 1-4 Nov 2017 & 18 females \\
\hline & & & Ksar Chellala & $35^{\circ} 15^{\prime} \mathrm{N}, 2^{\circ} 18^{\prime} \mathrm{E}$ & 21-22 Sep 2018 & 18 females \\
\hline & & & Hammadia & $35^{\circ} 26^{\prime} \mathrm{N}, 1^{\circ} 53^{\prime} \mathrm{E}$ & 16-19 Jun 2016 & 12 females \\
\hline & & & Hammadia & $35^{\circ} 26^{\prime} \mathrm{N}, 1^{\circ} 53^{\prime} \mathrm{E}$ & 1-4 Jul 2017 & 18 females \\
\hline & & & Hammadia & $35^{\circ} 26^{\prime} \mathrm{N}, 1^{\circ} 53^{\prime} \mathrm{E}$ & 7-8 Sep 2018 & 9 females \\
\hline & & & Rahouia & $35^{\circ} 29^{\prime} \mathrm{N}, 1^{\circ} 03^{\prime} \mathrm{E}$ & 23-26 Jun 2017 & 22 females \\
\hline & & & Rahouia & $35^{\circ} 29^{\prime} \mathrm{N}, 1^{\circ} 03^{\prime} \mathrm{E}$ & 9-12 Jul 2017 & 11 females \\
\hline & & & Rahouia & $35^{\circ} 29^{\prime} \mathrm{N}, 1^{\circ} \mathrm{O} 3^{\prime} \mathrm{E}$ & 21-22 Sep 2018 & 18 females \\
\hline & & & Machraa S'fa & $35^{\circ} 22^{\prime} \mathrm{N}, 1^{\circ} 03^{\prime} \mathrm{E}$ & 3-6 Jun 2017 & 12 females \\
\hline & & & Machraa S'fa & $35^{\circ} 22^{\prime} \mathrm{N}, 1^{\circ} 03^{\prime} \mathrm{E}$ & 23-26 Jun 2017 & 23 females \\
\hline & & & Machraa S'fa & $35^{\circ} 22^{\prime} \mathrm{N}, 1^{\circ} 03^{\prime} \mathrm{E}$ & 13-14 Jul 2018 & 7 females \\
\hline & & & Takhmaret & $35^{\circ} 06^{\prime} \mathrm{N}, 0^{\circ} 42^{\prime} \mathrm{E}$ & 5-8 Jul 2016 & 8 females \\
\hline & & & Takhmaret & $35^{\circ} 06^{\prime} \mathrm{N}, 0^{\circ} 42^{\prime} \mathrm{E}$ & 29 Jul-1 Aug 2017 & 6 females \\
\hline & & & Takhmaret & $35^{\circ} 06^{\prime} \mathrm{N}, 0^{\circ} 42^{\prime} \mathrm{E}$ & 8-9 Jun 2018 & 9 females \\
\hline & & \multirow[t]{2}{*}{ Egypt } & Sinaï, Khirba & $31^{\circ} 1^{\prime} \mathrm{N}, 32^{\circ} 53^{\prime} \mathrm{E}$ & 25 Apr 1979 & 10 females \\
\hline & & & Sinaï, Khirba & $31^{\circ} 1^{\prime} \mathrm{N}, 32^{\circ} 53^{\prime} \mathrm{E}$ & 24 Apr 1979 & 1 male \\
\hline
\end{tabular}


Table 1 (continued)

\begin{tabular}{|c|c|c|c|c|c|c|}
\hline Species & Type of material & Country & Locality & Coordinates & Date & Material examined \\
\hline \multirow{31}{*}{$\begin{array}{l}\text { Culicoides (Culicoides) grises- } \\
\text { cens Edwards }\end{array}$} & \multirow[t]{31}{*}{ Non-type material } & \multirow[t]{29}{*}{ Algeria } & Sougueur & $35^{\circ} 09^{\prime} \mathrm{N}, 1^{\circ} 31^{\prime} \mathrm{E}$ & 5-7 Apr 2017 & 10 females \\
\hline & & & Sougueur & $35^{\circ} 09^{\prime} \mathrm{N}, 1^{\circ} 31^{\prime} \mathrm{E}$ & 26-29 May 2017 & 13 females \\
\hline & & & Sougueur & $35^{\circ} 09^{\prime} \mathrm{N}, 1^{\circ} 31^{\prime} \mathrm{E}$ & 3-6 Jun 2017 & 5 females \\
\hline & & & Sougueur & $35^{\circ} 09^{\prime} \mathrm{N}, 1^{\circ} 31^{\prime} \mathrm{E}$ & 17-20 Jul 2017 & 13 females \\
\hline & & & Sougueur & $35^{\circ} 09^{\prime} \mathrm{N}, 1^{\circ} 31^{\prime} \mathrm{E}$ & 25-28 Jul 2017 & 11 females \\
\hline & & & Sougueur & $35^{\circ} 09^{\prime} \mathrm{N}, 1^{\circ} 31^{\prime} \mathrm{E}$ & 20-23 Sep 2017 & 15 females \\
\hline & & & Sougueur & $35^{\circ} 09^{\prime} \mathrm{N}, 1^{\circ} 31^{\prime} \mathrm{E}$ & 1-3 Oct 2017 & 12 females \\
\hline & & & Sougueur & $35^{\circ} 09^{\prime} \mathrm{N}, 1^{\circ} 31^{\prime} \mathrm{E}$ & 1-4 Jul 2018 & 9 females \\
\hline & & & Sougueur & $35^{\circ} 09^{\prime} \mathrm{N}, 1^{\circ} 31^{\prime} \mathrm{E}$ & 7-8 Sep 2018 & 9 females \\
\hline & & & Ain El Deheb & $34^{\circ} 51^{\prime} \mathrm{N}, 1^{\circ} 29^{\prime} \mathrm{E}$ & 29 Nov -5 Dec 2015 & 2 females \\
\hline & & & Ain El Deheb & $34^{\circ} 51^{\prime} \mathrm{N}, 1^{\circ} 29^{\prime} \mathrm{E}$ & 15-17 Sep 2016 & 15 females \\
\hline & & & Ain El Deheb & $34^{\circ} 51^{\prime} \mathrm{N}, 1^{\circ} 29^{\prime} \mathrm{E}$ & 5-7 Apr 2017 & 9 females \\
\hline & & & Ain El Deheb & $34^{\circ} 51^{\prime} \mathrm{N}, 1^{\circ} 29^{\prime} \mathrm{E}$ & 28-29 Sep 2018 & 11 females \\
\hline & & & Ksar Chellala & $35^{\circ} 15^{\prime} \mathrm{N}, 2^{\circ} 18^{\prime} \mathrm{E}$ & 26-29 May 2017 & 7 females \\
\hline & & & Ksar Chellala & $35^{\circ} 15^{\prime} \mathrm{N}, 2^{\circ} 18^{\prime} \mathrm{E}$ & 9-12 Nov 2017 & 6 females \\
\hline & & & Ksar Chellala & $35^{\circ} 15^{\prime} \mathrm{N}, 2^{\circ} 18^{\prime} \mathrm{E}$ & 21-22 Sep 2018 & 16 females \\
\hline & & & Hammadia & $35^{\circ} 26^{\prime} \mathrm{N}, 1^{\circ} 53^{\prime} \mathrm{E}$ & 15-17 Sep 2016 & 8 females \\
\hline & & & Hammadia & $35^{\circ} 26^{\prime} \mathrm{N}, 1^{\circ} 53^{\prime} \mathrm{E}$ & 28-30 Sep 2017 & 7 females \\
\hline & & & Hammadia & $35^{\circ} 26^{\prime} \mathrm{N}, 1^{\circ} 53^{\prime} \mathrm{E}$ & 1-3 Oct 2017 & 11 females \\
\hline & & & Hammadia & $35^{\circ} 26^{\prime} \mathrm{N}, 1^{\circ} 53^{\prime} \mathrm{E}$ & 28-29 Sep 2018 & 12 females \\
\hline & & & Rahouia & $35^{\circ} 29^{\prime} \mathrm{N}, 1^{\circ} 03^{\prime} \mathrm{E}$ & 24-27 Jun 2016 & 5 females \\
\hline & & & Rahouia & $35^{\circ} 29^{\prime} \mathrm{N}, 1^{\circ} 03^{\prime} \mathrm{E}$ & 12-15 Sep 2017 & 8 females \\
\hline & & & Rahouia & $35^{\circ} 29^{\prime} \mathrm{N}, 1^{\circ} 03^{\prime} \mathrm{E}$ & 21-22 Sep 2018 & 7 females \\
\hline & & & Machraa S'fa & $35^{\circ} 22^{\prime} \mathrm{N}, 1^{\circ} 03^{\prime} \mathrm{E}$ & 8-10 Sep 2016 & 9 females \\
\hline & & & Machraa S'fa & $35^{\circ} 22^{\prime} \mathrm{N}, 1^{\circ} 03^{\prime} \mathrm{E}$ & 26-29 May 2017 & 9 females \\
\hline & & & Machraa S'fa & $35^{\circ} 22^{\prime} \mathrm{N}, 1^{\circ} 03^{\prime} \mathrm{E}$ & 15-16 Jun 2018 & 7 females \\
\hline & & & Takhmaret & $35^{\circ} 06^{\prime} \mathrm{N}, 0^{\circ} 42^{\prime} \mathrm{E}$ & 20-23 Jun 2016 & 19 females \\
\hline & & & Takhmaret & $35^{\circ} 06^{\prime} \mathrm{N}, 0^{\circ} 42^{\prime} \mathrm{E}$ & 5-7 Apr 2017 & 10 females \\
\hline & & & Takhmaret & $35^{\circ} 06^{\prime} \mathrm{N}, 0^{\circ} 42^{\prime} \mathrm{E}$ & 28-29 Sep 2018 & 12 females \\
\hline & & \multirow[t]{2}{*}{ France } & Brognon & $49^{\circ} 55^{\prime} \mathrm{N}, 4^{\circ} 18^{\prime} \mathrm{E}$ & 2è-28 Jun 2007 & 1 female \\
\hline & & & Marcoux & $44^{\circ} 7^{\prime} \mathrm{N}, 6^{\circ} 17^{\prime} \mathrm{E}$ & 22-23 Jul 2009 & 4 females \\
\hline \multirow{9}{*}{$\begin{array}{l}\text { Culicoides (Culicoides) para- } \\
\text { doxalis Ramilo \& Delécolle }\end{array}$} & \multirow[t]{9}{*}{ Type material } & Algeria & Ain El Deheb & $34^{\circ} 51^{\prime} \mathrm{N}, 1^{\circ} 29^{\prime} \mathrm{E}$ & 27-28 Apr 2018 & 1 female \\
\hline & & \multirow[t]{8}{*}{ France, Corsica } & Pietra Corbara & $42^{\circ} 50^{\prime} \mathrm{N}, 9^{\circ} 26^{\prime} \mathrm{E}$ & 3-4 Jun 2003 & 1 female (holotype) \\
\hline & & & Pietra Corbara & $42^{\circ} 50^{\prime} \mathrm{N}, 9^{\circ} 26^{\prime} \mathrm{E}$ & 3-4 Jun 2003 & 2 female \\
\hline & & & Pietra Corbara & $42^{\circ} 50^{\prime} \mathrm{N}, 9^{\circ} 26^{\prime} \mathrm{E}$ & 6-7 Jul 2004 & 1 female \\
\hline & & & Porto Vecchio & $41^{\circ} 35^{\prime} \mathrm{N}, 9^{\circ} 15^{\prime} \mathrm{E}$ & 22-23 Sep 2005 & 2 females \\
\hline & & & Sarrola Carcopino & $42^{\circ} 0^{\prime} \mathrm{N}, 8^{\circ} 50^{\prime} \mathrm{E}$ & 26-27 Jun 2006 & 1 female \\
\hline & & & Sartene & $41^{\circ} 38^{\prime} \mathrm{N}, 8^{\circ} 57^{\prime} \mathrm{E}$ & 27-28 Sep 2005 & 1 female \\
\hline & & & Sartene & $41^{\circ} 38^{\prime} \mathrm{N}, 8^{\circ} 57^{\prime} \mathrm{E}$ & 12-13 Jun 2002 & 1 female \\
\hline & & & Sartene & $41^{\circ} 38^{\prime} \mathrm{N}, 8^{\circ} 57^{\prime} \mathrm{E}$ & 20-21 Jun 2002 & 1 female \\
\hline
\end{tabular}


Table 1 (continued)

\begin{tabular}{|c|c|c|c|c|c|c|}
\hline Species & Type of material & Country & Locality & Coordinates & Date & Material examined \\
\hline \multirow{7}{*}{$\begin{array}{c}\text { Culicoides (Sensiculicoides) } \\
\text { shaklawensis Khalaf }\end{array}$} & \multirow[t]{7}{*}{ Non-type material } & \multirow[t]{4}{*}{ Algeria } & Tiaret & $35^{\circ} 23^{\prime} \mathrm{N}, 1^{\circ} 21^{\prime} \mathrm{E}$ & 9-14 Mar 2016 & 1 female \\
\hline & & & Tiaret & $35^{\circ} 23^{\prime} \mathrm{N}, 1^{\circ} 21^{\prime} \mathrm{E}$ & 15-16 Jun 2018 & 1 female \\
\hline & & & Tiaret & $35^{\circ} 23^{\prime} \mathrm{N}, 1^{\circ} 21^{\prime} \mathrm{E}$ & 27-28 Jul 2018 & 1 female \\
\hline & & & Bougara & $35^{\circ} 29^{\prime} \mathrm{N}, 1^{\circ} 55^{\prime} \mathrm{E}$ & 29-31 Oct 2016 & 1 female \\
\hline & & \multirow[t]{3}{*}{ France } & Calvi & $42^{\circ} 32^{\prime} \mathrm{N}, 8^{\circ} 45^{\prime} \mathrm{E}$ & 23-24 Jun 2003 & 1 female \\
\hline & & & Marcoux & $44^{\circ} 7^{\prime} \mathrm{N}, 6^{\circ} 17^{\prime} \mathrm{E}$ & 22-23 Jul 2009 & 1 female \\
\hline & & & Moltifao & $42^{\circ} 28^{\prime} \mathrm{N}, 9^{\circ} 7^{\prime} \mathrm{E}$ & 5-6 Aug 2003 & 1 female \\
\hline \multirow{5}{*}{$\begin{array}{l}\text { Culicoides (Sensiculicoides) } \\
\text { simulator Edwards }\end{array}$} & \multirow[t]{5}{*}{ Non-type material } & Algeria & Tiaret & $35^{\circ} 23^{\prime} \mathrm{N}, 1^{\circ} 21^{\prime} \mathrm{E}$ & 1-2 Jun 2018 & 1 female \\
\hline & & \multirow[t]{4}{*}{ France } & Aleria & $42^{\circ} 6^{\prime} \mathrm{N}, 9^{\circ} 29^{\prime} \mathrm{E}$ & 8-9 Jun 2004 & 1 female \\
\hline & & & La Chapelle d'Andaine & $48^{\circ} 31^{\prime} \mathrm{N}, 0^{\circ} 28^{\prime} \mathrm{W}$ & 19-20 Jun 2012 & 1 female \\
\hline & & & Marcoux & $44^{\circ} 7^{\prime} \mathrm{N}, 6^{\circ} 17^{\prime} \mathrm{E}$ & 22-23 Jul 2009 & 1 female \\
\hline & & & Remilly Aillicourt & $49^{\circ} 39^{\prime} \mathrm{N}, 4^{\circ} 58^{\prime} \mathrm{E}$ & 24-25 May 2007 & 1 female \\
\hline \multirow{8}{*}{$\begin{array}{l}\text { Culicoides (Sensiculicoides) } \\
\text { univittatus Vimmer }\end{array}$} & \multirow[t]{8}{*}{ Non-type material } & \multirow[t]{3}{*}{ Algeria } & Tiaret & $35^{\circ} 23^{\prime} \mathrm{N}, 1^{\circ} 21^{\prime} \mathrm{E}$ & 10-12 Dec 2016 & 1 male, 1 female \\
\hline & & & Tiaret & $35^{\circ} 23^{\prime} \mathrm{N}, 1^{\circ} 21^{\prime} \mathrm{E}$ & 15-17 Mar 2017 & 4 males, 7 females \\
\hline & & & Tiaret & $35^{\circ} 23^{\prime} \mathrm{N}, 1^{\circ} 21^{\prime} \mathrm{E}$ & 4-5 May 2018 & 2 females \\
\hline & & \multirow[t]{5}{*}{ France, Corsica } & Figari & $41^{\circ} 30^{\prime} \mathrm{N}, 9^{\circ} 5^{\prime} \mathrm{E}$ & 10-11 Apr 2006 & 1 male, 4 females \\
\hline & & & Figari & $41^{\circ} 30^{\prime} \mathrm{N}, 9^{\circ} 5^{\prime} \mathrm{E}$ & 14-15 Feb 2011 & 1 female \\
\hline & & & Moltifao & $42^{\circ} 28^{\prime} \mathrm{N}, 9^{\circ} 7^{\prime} \mathrm{E}$ & 25-26 Apr 2005 & 1 female \\
\hline & & & San Giuliano & $42^{\circ} 17^{\prime} \mathrm{N}, 9^{\circ} 32^{\prime} \mathrm{E}$ & 6-7 Mar 2003 & 2 males \\
\hline & & & San Giuliano & $42^{\circ} 17^{\prime} \mathrm{N}, 9^{\circ} 32^{\prime} \mathrm{E}$ & 10-11 Feb 2011 & 1 female \\
\hline \multirow{5}{*}{$\begin{array}{c}\text { Culicoides (Silvaticulicoides) } \\
\text { achrayi Kettle \& Lawson }\end{array}$} & \multirow[t]{5}{*}{ Non-type material } & \multirow[t]{3}{*}{ Algeria } & Hammadia & $35^{\circ} 26^{\prime} \mathrm{N}, 1^{\circ} 53^{\prime} \mathrm{E}$ & 27-28 Apr 2018 & 1 female \\
\hline & & & Sougueur & $35^{\circ} 09^{\prime} \mathrm{N}, 1^{\circ} 31^{\prime} \mathrm{E}$ & 15-17 Oct 2016 & 1 female \\
\hline & & & Sougueur & $35^{\circ} 09^{\prime} \mathrm{N}, 1^{\circ} 31^{\prime} \mathrm{E}$ & 5-7 Apr 2017 & 1 female \\
\hline & & \multirow[t]{2}{*}{ France } & Crozon & $48^{\circ} 16^{\prime} \mathrm{N}, 4^{\circ} 30^{\prime} \mathrm{W}$ & 13-14 Jun 2011 & 2 females \\
\hline & & & Pontivy & $48^{\circ} 4^{\prime} \mathrm{N}, 2^{\circ} 58^{\prime} \mathrm{W}$ & 18-19 Jan 2012 & 3 females \\
\hline \multirow{7}{*}{$\begin{array}{l}\text { Culicoides (Silvaticulicoides) } \\
\text { picturatus Kremer \& Deduit }\end{array}$} & \multirow{7}{*}{ Non-type material } & \multirow[t]{3}{*}{ Algeria } & Tiaret & $35^{\circ} 23^{\prime} \mathrm{N}, 1^{\circ} 21^{\prime} \mathrm{E}$ & 24-27 Jun 2016 & 1 female, 1 male \\
\hline & & & Tiaret & $35^{\circ} 23^{\prime} \mathrm{N}, 1^{\circ} 21^{\prime} \mathrm{E}$ & 10-12 Jul 2016 & 1 female \\
\hline & & & Tiaret & $35^{\circ} 23^{\prime} \mathrm{N}, 1^{\circ} 21^{\prime} \mathrm{E}$ & 28-29 Sep 2018 & 1 female \\
\hline & & \multirow[t]{4}{*}{ France } & Humes Jorquenay & $47^{\circ} 54^{\prime} \mathrm{N}, 5^{\circ} 18^{\prime} \mathrm{E}$ & 6-7 Jun 2011 & 1 male \\
\hline & & & Lazer & $44^{\circ} 21^{\prime} \mathrm{N}, 5^{\circ} 51^{\prime} \mathrm{E}$ & 18-19 Apr 2011 & 1 female \\
\hline & & & Porto Vecchio & $41^{\circ} 35^{\prime} \mathrm{N}, 9^{\circ} 15^{\prime} \mathrm{E}$ & 29-30 May 2002 & 2 males \\
\hline & & & Saignon & $43^{\circ} 51^{\prime} \mathrm{N}, 5^{\circ} 27^{\prime} \mathrm{E}$ & 6-7 Jun 2011 & 3 females \\
\hline
\end{tabular}

\section{Author details}

${ }^{1}$ Ecole Nationale Supérieure de Biotechnologie, Taoufk Khaznadar, nouveau pôle universitaire Ali Mendjeli, B.P. E66, 25100, Constantine, Algérie. ${ }^{2}$ Faculté des Sciences de la Nature et de la Vie, Université Ibn Khaldoun, B.P.75 Zaaroura, Tiaret 1400, Algérie. ${ }^{3}$ Laboratoire de Biosystématique et Ecologie des Arthropodes, Faculté des Sciences de la Nature et de la Vie, Département de Biologie Animale, Université Frères Mentouri, Constantine 1 2500, Algérie. ${ }^{4}$ CIRAD, UMR ASTRE, 34398 Montpellier, France. Institut de Parasitologie et de Pathologies Tropicales de Strasbourg (IPPTS), UR 7292, 3 Rue Koeberlé, 67000 Strasbourg, France. ${ }^{6}$ ASTRE, University of Montpellier, CIRAD, INRAE, Montpellier, France. ${ }^{7}$ CIRAD, UMR ASTRE, Sainte-Clotilde, 97491 La Réunion, France. ${ }^{8}$ CIRAD, UMR ASTRE, 10101 Rabat, Morocco. ${ }^{9}$ Institut Agronomique et Vétérinaire Hassan II, Unité Microbiologie, Immunologie et Maladies Contagieuses, 10100 Rabat, Morocco.

Published online: 12 July 2021

\section{Reference}

1. Belkharchouche M, Berchi S, Mathieu B, Rakotoarivony I, Duhayon M, Baldet T, Balenghien T. Update of the Culicoides (Diptera: Ceratopogonidae) species checklist from Algeria with 10 new records. Parasites Vectors. 2020;13:463. https://doi.org/10.1186/s13071-020-04335-4.

\section{Publisher's Note}

Springer Nature remains neutral with regard to jurisdictional claims in published maps and institutional affiliations. 